${ }^{1}$ Departamento de Ciencias de la Salud. Facultad de Medicina, Pontificia Universidad Católica de Chile. Santiago Chile

${ }^{2}$ Departamento de Cirugía Digestiva. Facultad de Medicina, Pontificia Universidad Católica de Chile. Santiago Chile.

${ }^{3}$ Departamento de Medicina Interna, Facultad de Medicina, Pontificia Universidad Católica de Chile. Santiago, Chile.

4Departamento de Gastroenterología, Facultad de Medicina, Pontificia Universidad Católica de Chile. Santiago, Chile.

Departamento de Enfermedades Respiratorias. Facultad de Medicina Pontificia Universidad Católica de Chile. Santiago, Chile.

${ }^{6}$ Departamento de Anestesia, Facultad de Medicina, Pontificia Universidad Católica de Chile. Santiago, Chile.

Escuela Medicina. Facultad de Medicina, Pontificia Universidad Católica de Chile. Santiago, Chile.

Departamento de Salud Pública. Facultad de Medicina, Pontificia Universidad Católica de Chile. Santiago, Chile.

${ }^{9}$ Centro de Cirugía Experimental y Simulación. Facultad de Medicina

Pontificia Universidad Católica de Chile. Santiago, Chile.

${ }^{10}$ Centro de Educación Médica. Facultad de Medicina, Pontificia Universidad Católica de Chile. Santiago, Chile. ${ }^{a}$ Kinesiólogo. ${ }^{\text {bMédico. }}$ 'Bioquímico. ¿Estadístico. eFonoaudiólogo.

Artículo financiado por proyecto FONDEDOC 2016 A. R. Estandarización de curva de aprendizaje y transferencia a pacientes reales de habilidades en paracentesis adquiridas mediante simulación y FONDEDOC 2016 I.L.

Evaluación de la mantención a largo plazo de la competencia en la ejecución de la toracocentesis en ambiente simulado en alumnos de medicina

Recibido el 13 de octubre de 2017 aceptado el 31 de mayo de 2018

Correspondencia a: Ignacio Villagrán Gutiérrez Departamento de Ciencias de la Salud Dirección: Vicuña Mackenna 4860 Macul, Santiago, Chile. invillagran@uc.cl

\section{Percepción de estudiantes de pregrado de Medicina de talleres de simulación de procedimientos médico-quirúrgicos}

\author{
IGNACIO VILLAGRÁN ${ }^{1, \mathrm{a}}$, RODRIGO TEJOS $2,9, \mathrm{~b}$, \\ JAVIER CHAHUAN ${ }^{3, b}$, THOMAS USLAR ${ }^{3, b}$, \\ MARGARITA PIZARRO ${ }^{4, c}$, JULIÁN VARAS ${ }^{2,9, b}$, \\ PABLO ACHURRA ${ }^{2,9, b}$, ISABEL LEIVA $^{5, b}$, CLAUDIO NAZAR ${ }^{6, b}$, \\ MARISOL SIRHAN ${ }^{4,10, \mathrm{~b}}$, JAVIER URIBE ${ }^{7, \mathrm{~b}}$, CRISTIAN RUZ $^{7, \mathrm{~b}}$, \\ CARLOS VILLAFRANCA ${ }^{7, b}$, ROMINA SOZA ${ }^{3, b}$, NANCY SOLÍS $^{4, c}$, \\ EDUARDO FUENTES-LÓPEZ ${ }^{1, \mathrm{e}}$, OSLANDO PADILLA ${ }^{8, \mathrm{~d},}$ \\ MARCIA CORVETTO ${ }^{6,9, b}$, ARNOLDO RIQUELME ${ }^{1,4,10, b}$

\section{Undergraduate student's perception of clinical simulation workshops: assessment of an instrument}

Background: Simulation is a useful training tool for undergraduate medical students. A valid instrument is needed to assess students' perception of simulation workshops. Aim: To adapt and validate an instrument to assess the undergraduate medical student's perception of simulation workshops of clinical procedures. Material and Methods: Delphi Methodology was used to adapt the instrument. Exploratory and confirmatory analyses were performed to determine the construct validity and Cronbach's Alpha (0 to 1) for internal consistency of the instrument. Results: A Delphi panel of 10 experts adapted a seven-item questionnaire (Likert scale 1-5; ranging from 7 to 35) and four open-questions. After 3-delphi-rounds, the instrument was administered to 210 students in six simulation training programs (Paracentesis, Cardiopulmonary Resuscitation, Airway management, Sutures, Thoracentesis and Nursing Procedures). The instrument was considered unidimensional in the factorial analysis. The overall median (Q1-Q3) score was 34 ranging from 32 to 35 and the Cronbach Alpha coefficient was 0.72, indicating a good reliability. Conclusions: The perception questionnaire is a useful and reliable instrument to assess students' perceptions of clinical simulations.

(Rev Med Chile 2018; 146: 786-795)

Key words: Education; Education, Medical; Clinical Competence; Students, Medical; Surveys and Questionnaires.
E xiste una creciente necesidad de minimizar posibles errores en la práctica médica, por lo que han surgido estrategias para disminuir el potencial impacto negativo del entrenamiento de estudiantes en la seguridad de los pacientes ${ }^{1}$. En Chile, las Escuelas de Medicina han realizado reformas curriculares en pregrado, incorporando conceptos innovadores en educación médica, con 
el fin de mejorar los procesos de enseñanza-aprendizaje. La Escuela de Medicina de la Pontificia Universidad Católica de Chile (EMPUC), establece como uno de los ejes de su reforma curricular, "Utilizar metodologías tendientes a favorecer la autonomía en los aprendizajes y la participación activa de los estudiantes en su formación personal y el desarrollo del profesionalismo"2. Dentro de las nuevas estrategias implementadas, la simulación ha cobrado relevancia, creando un ambiente ideal para el aprendizaje con actividades diseñadas para ser predecibles, consistentes, estandarizadas, seguras y reproducibles ${ }^{3,4}$. En salud, la simulación sitúa a un estudiante en un contexto que imite algún aspecto de la realidad clínica. Esta metodología complementa el modelo tradicional, acortando las curvas de aprendizaje y disminuyendo los riesgos asociados al procedimiento ${ }^{5,6}$.

Esta estrategia, se ha vuelto fundamental en cursos de pregrado de EMPUC, reportándose una adquisición temprana de habilidades técnicas de procedimientos, acercándose a nivel de expertos, con la posibilidad de equivocarse y aprender del error, sin riesgos para el paciente ni para el alumno ${ }^{7-10}$. Este modelo, ha sido empleado en diversos procedimientos, desde punciones venosas, suturas, punciones lumbares y cricotiroidotomías, hasta otros más complejos como anastomosis intestinales laparoscópicas ${ }^{11,12}$.

Como complemento, los modelos simulados permiten evaluaciones estandarizadas, otorgando una metodología eficiente para la adquisición de competencias médico-quirúgicas y transversales necesarias en la práctica clínica ${ }^{13-17}$. La evaluación en simulación, se ha centrado en medir el desempeño de los alumnos ejecutando las tareas que se pretenden enseñar. Para esto se han utilizado parámetros como: tiempo de ejecución, cantidad de movimientos realizados (medido con sistema del Imperial College Surgical Assessment DeviceICSAD) y escalas de evaluación por observación directa como la pauta OSATS (Objective Surgical Assessment of Technical Skills $)^{18}$.

Aunque la simulación ha demostrado buenos resultados, un problema emergente es la estandarización de la evaluación de la percepción de los estudiantes sobre su aprendizaje en escenarios simulados de procedimientos médico-quirúrgicos, siendo éste un factor clave para la mejora continua de las actividades de simulación que forman parte de los programas curriculares de pregrado.
En la literatura existen escasas experiencias sobre evaluación de percepción en talleres simulados, siendo además enfocadas en postgrado. Wayne et al. utilizó una encuesta para conocer la percepción de un taller de simulación de Reanimación Cardíaca Avanzada ${ }^{19}$, Barsuk et al. usó una pauta para evaluación de la percepción de estudiantes de postgrado en un taller simulado de los procedimientos de: Toracocentesis ${ }^{20}$, Inserción de Catéter de Hemodiálisis ${ }^{21}$ y Paracentesis ${ }^{22}$. Ambos estudios carecen de evaluación de las propiedades psicométricas de los instrumentos utilizados.

Pese a la utilidad de este tipo de instrumentos para la educación médica, no se ha encontrado a la fecha, información respecto a la validez y confiabilidad de los instrumentos aplicados que permitan estandarizar el proceso de medición de la percepción de los estudiantes en estos talleres simulados de pregrado. Dada la creciente incorporación de la simulación como herramienta educacional, existe la necesidad de evaluar no sólo el desempeño de los alumnos sino también la percepción que éstos tienen de su entrenamiento. Así, el objetivo de este trabajo fue adaptar y validar un instrumento para evaluar la percepción de los estudiantes de los talleres de simulación de destrezas clínicas de procedimientos en pregrado de Medicina.

\section{Métodos}

\section{Diseño de la encuesta de percepción}

Se realizó una búsqueda en la literatura sobre escalas de evaluación para la percepción de procedimientos simulados ${ }^{19-22}$. Utilizando como base la encuesta generada por Barsuk ${ }^{22}$, se creó una nueva versión para cumplir con criterios de validez de contenido y muestreo de las actividades docentes relacionadas con talleres de simulación de pregrado. Un panel de expertos local seleccionó las preguntas a incluir en la encuesta mediante metodología Delfi modificada ${ }^{23}$.

\section{Participantes y aplicación}

La encuesta fue piloteada en estudiantes del taller de paracentesis y, posteriormente, aplicada a estudiantes de tercer y cuarto año de pregrado de EMPUC en 6 programas de entrenamiento de destrezas en procedimientos, entre octubre de 2015 y enero de 2017. Todos los talleres se realizan en el Centro de Simulación UC y consisten en la 
práctica de los distintos procedimientos con un modelo de simulación validado. El entrenamiento es guiado por tutores expertos quienes evalúan el desempeño de los alumnos mediante una pauta de observación directa, entregando retroalimentacion inmediata a los estudiantes. Los tutores son miembros del equipo de Simulación UC capacitados para la enseñanza con este tipo de metodología, existiendo un docente a cargo de las rotaciones quien coordina los tutores expertos para el entrenamiento.

Se utilizó el método propuesto por MacCallum, Browne y Sugawara ${ }^{24-26}$, se estima en 205 los sujetos necesarios poder evaluar estadísticamente la hipótesis de que el modelo factorial tiene al menos ajuste moderado.

El protocolo fue aprobado por el comité de ética de nuestra institución y los datos fueron entregados y utilizados en forma anónima.

\section{Análisis estadístico}

Los indicadores de validez de constructo se obtuvieron mediante análisis exploratorio y confirmatorio de factores. El análisis exploratorio permitió detectar la existencia de variables latentes ${ }^{27,28}$ y el análisis confirmatorio el número de factores latentes y su estructura en los datos ${ }^{27}$. Se realizó un análisis de confiabilidad mediante el Coeficiente de Alfa de Cronbach, considerando $<0,4$ baja, 0,4-0,59 moderada, $0,6-0,8$ buena y $>0,8$ excelente confiabilidad ${ }^{29,30}$.

El análisis descriptivo y analítico de los resultados se realizó mediante un test de proporciones. Se aplicó el test de comparaciones pareadas de Fisher, ajustando por contrastes múltiples ("Bonferroni”). Se determinaron los estadígrafos descriptivos para los puntajes de cada pregunta y el puntaje global para cada taller.

\section{Resultados}

\section{Diseño de la encuesta de percepción}

En base a la búsqueda, se identificó un instrumento de evaluación diseñado para medir la percepción de talleres simulados de paracentesis de estudiantes de postgrado ${ }^{22}$ y se adaptó con el fin de aplicarla a pregrado de EMPUC. Un panel de expertos $(n=10)$ refinó la redacción de los ítems y las preguntas abiertas mediante técnica Delfi modificada de 3 rondas. Este panel de expertos local estaba compuesto por 2 Anestesiólogos (C.N., M.C.), 1 Cirujano (J.V.), 2 Bioquímicos (M.P., N.S.), 2 Gastroenterólogos (M.S., A.R.), 1 Estadístico (O.P.), 1 Broncopulmonar (I.L.) y 1 Residente de Cirugía Digestiva (P.A.)

Se definió una encuesta con escala Likert de 7 ítems, a partir de publicación de Barsuk y cols. ${ }^{22}$, los cuales fueron traducidos al español y traducción reversa, ajustados y aplicados en talleres de simulación de procedimientos médico-quirúrgicos en pregrado.

\section{Estadística descriptiva y analítica}

Se encuestaron 210 alumnos de 6 programas de entrenamiento por simulación: Paracentesis (67), Reanimación Cardiopulmonar (RCP) (18), Vía Aérea (19), Suturas (41), Toracocentesis (51) y Procedimientos de Enfermería (14).

Debido a que las respuestas a las preguntas de la escala constituyen variables ordinales se estimaron proporciones con sus intervalos de confianza. Destacó la variabilidad de las aseveraciones 2, 3, 4 y 5, en relación a los ítems 1,6 y 7 . Todas las comparaciones entre las respuestas de la aseveración 1 fueron significativas $(\mathrm{p}<0,001)$. Algo similar ocurrió en las respuestas a las afirmaciones 6 y 7 . En las respuestas de la aseveración 2, los contrastes con diferencias significativas $(\mathrm{p}<0,001)$ se observaron entre opciones con mayor preferencia (de acuerdo y completamente de acuerdo), y entre cada una de estas y el resto con menor preferencia $(\mathrm{p}<0,001)$. En las afirmaciones 3 y 5 existieron diferencias significativas al comparar preferencias $(\mathrm{p}<0,001)$ a excepción de los contrastes entre afirmaciones de menor preferencia (en desacuerdo y no está seguro o no tiene opinión). En la afirmación 4 existieron diferencias al comparar sus preferencias $(\mathrm{p}<0,001)$ a excepción de los contrastes entre afirmaciones de menor preferencia (en desacuerdo y no está seguro o no tiene opinión), y entre las dos de mayor preferencia (de acuerdo y completamente de acuerdo) (Tabla 1). Los resultados cualitativos de las preguntas abiertas no se analizaron para este trabajo.

\section{Puntajes globales}

Se estiman los estadígrafos descriptivos para el puntaje por pregunta (Tabla 2) y global según el taller realizado (Figura 1). Se determinó la mediana y percentil 25-75 (Q1-Q3). Los estudiantes evaluaron positivamente los talleres: paracentesis 
Tabla 1. Proporciones para las respuestas de cada ítem de la encuesta de percepción’a

\begin{tabular}{|c|c|c|c|c|c|}
\hline Aseveraciones & $\begin{array}{l}\text { Completa- } \\
\text { mente en } \\
\text { desacuerdo }\end{array}$ & $\begin{array}{c}\text { En } \\
\text { desacuerdo }\end{array}$ & $\begin{array}{l}\text { No está } \\
\text { seguro o } \\
\text { no tiene } \\
\text { opinión }\end{array}$ & De acuerdo & $\begin{array}{l}\text { Completa- } \\
\text { mente de } \\
\text { acuerdo }\end{array}$ \\
\hline $\begin{array}{l}\text { 1. La práctica de modelos simulados mejora mis } \\
\text { destrezas relacionadas con la realización de } \\
\text { este procedimiento }\end{array}$ & $\begin{array}{l}\operatorname{Sin} \\
\text { observaciones }\end{array}$ & $\begin{array}{l}\text { Sin } \\
\text { observaciones }\end{array}$ & $\begin{array}{c}0,48 \\
(0,07-3,35)\end{array}$ & $\begin{array}{c}12,86 \\
(8,94-18,15)\end{array}$ & $\begin{array}{c}86,40 \\
(81,32-90,66)\end{array}$ \\
\hline $\begin{array}{l}\text { 2. Recibí retroalimentación educacional útil en } \\
\text { la(s) sesión(es) de entrenamiento }\end{array}$ & $\begin{array}{c}0,95 \\
(0,24-3,77)\end{array}$ & $\begin{array}{c}0,48 \\
(0,07-3,35)\end{array}$ & $\begin{array}{c}1,91 \\
(0,71-5,00)\end{array}$ & $\begin{array}{c}16,19 \\
(11,77-21,86)\end{array}$ & $\begin{array}{c}80,47 \\
(74,50-85,33)\end{array}$ \\
\hline $\begin{array}{l}\text { 3. La práctico con el modelo permite cometer } \\
\text { errores que probablemente sucedan en el } \\
\text { ambiente clínico real }\end{array}$ & $\begin{array}{l}\operatorname{Sin} \\
\text { observaciones }\end{array}$ & $\begin{array}{c}2,38 \\
(0,99-5,79)\end{array}$ & $\begin{array}{c}0,48 \\
(0,07-3,35)\end{array}$ & $\begin{array}{c}21,43 \\
(16,36-27,55)\end{array}$ & $\begin{array}{c}75,71 \\
(69,40-81,08)\end{array}$ \\
\hline $\begin{array}{l}\text { 4. El modelo usado en el taller simula o repre- } \\
\text { senta el procedimiento de manera realista }\end{array}$ & $\begin{array}{l}\operatorname{Sin} \\
\text { observaciones }\end{array}$ & $\begin{array}{c}5,24 \\
(2,91-9,25)\end{array}$ & $\begin{array}{c}7,14 \\
(4,33-11,55)\end{array}$ & $\begin{array}{c}43,81 \\
(37,20-50,65)\end{array}$ & $\begin{array}{c}45,81 \\
(37,20-50,65)\end{array}$ \\
\hline $\begin{array}{l}\text { 5. La práctica de procedimientos con el modelo } \\
\text { simulado refuerza mi confianza en relación a } \\
\text { mis destrezas clínicas }\end{array}$ & $\begin{array}{l}\operatorname{Sin} \\
\text { observaciones }\end{array}$ & $\begin{array}{c}0,95 \\
(0,02-3,77)\end{array}$ & $\begin{array}{c}0,95 \\
(0,02-3,77)\end{array}$ & $\begin{array}{c}18,57 \\
(13,84-24,46)\end{array}$ & $\begin{array}{c}79,52 \\
(73,48-84,48)\end{array}$ \\
\hline $\begin{array}{l}\text { 6. Los talleres prácticos, usando modelos de pro- } \\
\text { cedimientos simulados, deberían ser un compo- } \\
\text { nente obligatorio en la enseñanza de Medicina }\end{array}$ & $\begin{array}{l}\operatorname{Sin} \\
\text { observaciones }\end{array}$ & $\begin{array}{c}\text { Sin } \\
\text { observaciones }\end{array}$ & $\begin{array}{c}0,48 \\
(0,07-3,35)\end{array}$ & $\begin{array}{c}7,14 \\
(4,33-11,55)\end{array}$ & $\begin{array}{c}92,38 \\
(87,88-95,30)\end{array}$ \\
\hline $\begin{array}{l}\text { 7. La práctica con modelos de simulación me } \\
\text { ayuda a prepararme para realizar procedi- } \\
\text { mientos clínicos de mejor manera que sólo } \\
\text { con la experiencia clínica exclusiva }\end{array}$ & $\begin{array}{l}\operatorname{Sin} \\
\text { observaciones }\end{array}$ & $\begin{array}{c}\text { Sin } \\
\text { observaciones }\end{array}$ & $\begin{array}{c}0,48 \\
(0,07-3,35)\end{array}$ & $\begin{array}{c}10,48 \\
(6,97-15,44)\end{array}$ & $\begin{array}{c}89,05 \\
(84,00-92,64)\end{array}$ \\
\hline
\end{tabular}

a Proporciones con sus respectivos intervalos de confianza al 95\%.

Tabla 2. Medianas para las respuestas de cada ítem

\begin{tabular}{|lc|}
\hline Aseveraciones & Mediana \\
\hline $\begin{array}{lc}\text { La práctica de modelos simulados mejora mis destrezas relacionadas con la realización de este procedimiento } \\
\text { Recibí retroalimentación educacional útil en la(s) sesión(es) de entrenamiento }\end{array}$ & 5 \\
\hline $\begin{array}{l}\text { La práctica con el modelo permite cometer errores que probablemente sucedan en el ambiente clínico real } \\
\text { El modelo usado en el taller simula o representa el procedimiento de manera realista }\end{array}$ & 5 \\
\hline $\begin{array}{l}\text { La práctica de procedimientos con el modelo simulado refuerza mi confianza en relación a mis destrezas clínicas } \\
\text { Los talleres prácticos, usando modelos de procedimientos simulados, deberían ser un componente obligatorio } \\
\text { en la enseñanza de Medicina }\end{array}$ & 5 \\
$\begin{array}{l}\text { La práctica con modelos de simulación me ayuda a prepararme para realizar procedimientos clínicos de mejor } \\
\text { manera que sólo con la experiencia clínica exclusiva }\end{array}$ & 5 \\
\hline
\end{tabular}

(mediana 34; 32-35), rcp (mediana 35; 32-35), suturas (mediana 33; 31-34), toracocentesis (mediana 34 ; 33-35), vía aérea (mediana 33; 31-34) y procedimientos de enfermería (mediana 34; 33-35). La mediana del puntaje global fue de 34 $(32-35)$.

\section{Análisis exploratorio de factores}

La matriz se construyó en base a correlaciones policóricas ${ }^{31}$, destacando que pocas se encontraron por debajo de 0,3 o sobre 0,90 . Luego, se extrajeron los factores y las cargas de cada una de las aseveraciones de la encuesta. Se constató que existe un 


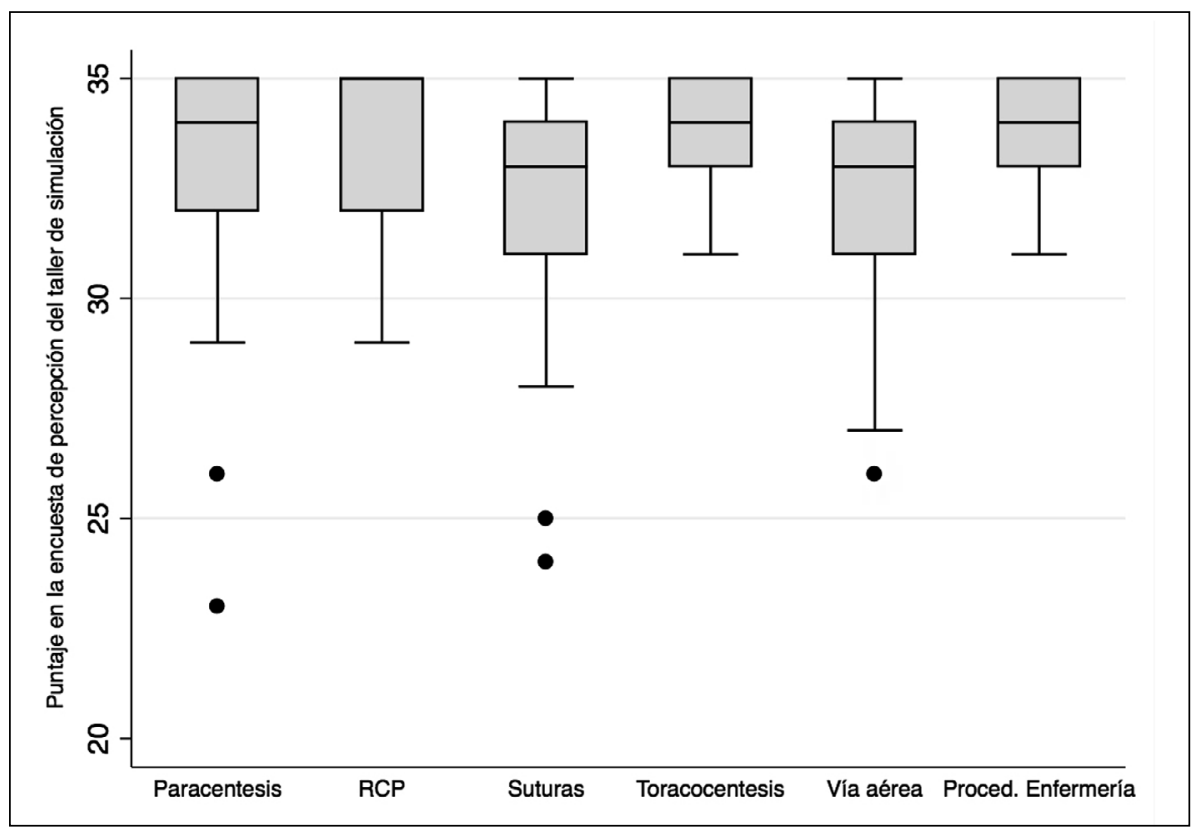

Figura 1. Medianas y rangos intercuartiles de puntajes globales en la encuesta según cada taller de simulación realizado.

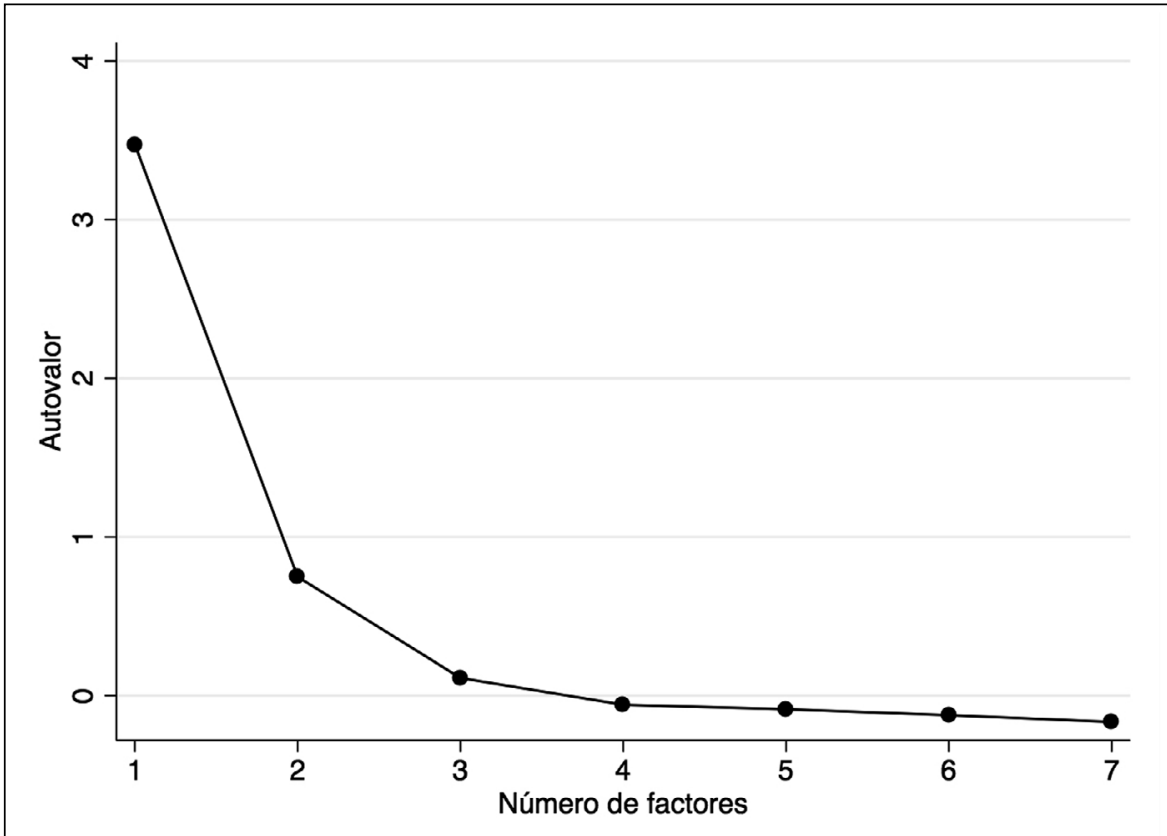

Figura 2. Gráfico de sedimentación de la matriz de varianza-covarianza.

factor con autovalor sobre uno $(3,47)$, siendo 4,64 veces mayor al segundo factor (Figura 2). Dicho factor explica $89,1 \%$ de la varianza de las puntuaciones. Todas las cargas son elevadas $(>0,4), y$ no existen marcada representación en más de un factor (Tabla 3). El índice "Kaiser-Meyer-Olkin" que cuantifica si la muestra para cada aseveración es adecuada, en su mayoría se encontró entre 0,70 y 0,91 (muestra "adecuada" a "excelente") 32 .

\section{Análisis confirmatorio de factores}

A través del análisis confirmatorio se estimó 
Tabla 3. Cargas factoriales e índice de Kaiser-Meyer-Olkin (KMO) de las preguntas incluidas en la "Encuesta de percepción de los estudiantes del taller de simulación de procedimientos"

\begin{tabular}{|c|c|c|}
\hline Aseveraciones & $\begin{array}{l}\text { Carga } \\
\text { factorial }\end{array}$ & KMO \\
\hline $\begin{array}{l}\text { La práctica de modelos simulados mejora mis destrezas relacionadas con la realización de este } \\
\text { procedimiento }\end{array}$ & 0,619 & 0,928 \\
\hline Recibí retroalimentación educacional útil en la(s) sesión(es) de entrenamiento & 0,672 & 0,928 \\
\hline $\begin{array}{l}\text { La práctica con el modelo permite cometer errores que probablemente sucedan en el ambiente } \\
\text { clínico real }\end{array}$ & 0,549 & 0,743 \\
\hline El modelo usado en el taller simula o representa el procedimiento de manera realista & 0,780 & 0,828 \\
\hline $\begin{array}{l}\text { La práctica de procedimientos con el modelo simulado refuerza mi confianza en relación a mis } \\
\text { destrezas clínicas }\end{array}$ & 0,724 & 0,896 \\
\hline $\begin{array}{l}\text { Los talleres prácticos, usando modelos de procedimientos simulados, deberían ser un componente } \\
\text { obligatorio en la enseñanza de Medicina }\end{array}$ & 0,704 & 0,678 \\
\hline $\begin{array}{l}\text { La práctica con modelos de simulación me ayuda a prepararme para realizar procedimientos clínicos } \\
\text { de mejor manera que sólo con la experiencia clínica exclusiva }\end{array}$ & 0,842 & 0,734 \\
\hline
\end{tabular}

el $R^{2}$, cuantificando el porcentaje de varianza de la aseveración que es explicada por el factor identificado ${ }^{33}$. Las aseveraciones obtuvieron un coeficiente superior a 0,3 , siendo estadísticamente significativo. Se utilizó como estimador Weighted Least Squares Mean Variance adjusted, que es pertinente para variables categóricas ${ }^{28,33}$. Diversos índices de bondad de ajuste fueron obtenidos: (a) Comparative fit index (CFI) que se relaciona con el grado de correlación presente entre las preguntas del cuestionario; (b) Índice de ajuste Tucker-Lewis (TLI) que penaliza modelos complejos ${ }^{28}$; y (c) el índice RSMEA que cuantifica falta de ajuste del modelo ${ }^{28}$. Se evaluó el ajuste del modelo en relación al RSMEA $\left(\mathrm{H}_{0}\right.$ : RMSEA $\left.\leq 0,05\right)$. Los resultados del análisis exploratorio y confirmatorio permiten concluir que se trata de un instrumento de 7 ítems unidimensional.

El modelo propuesto inicialmente si bien mostró adecuados valores para los índices CFI y TLI, el test "Close fit" fue estadísticamente significativo $(p=0,005)$, rechazando la hipótesis de ajuste adecuado. En base al índice de modificación se deja libre de error de covarianza las preguntas 3 y 4 . El modelo final propuesto mostró un $\mathrm{CFI}=0,971$, siendo mayor al punto de corte propuesto por $\mathrm{Hu}$ y Bentler ${ }^{34,35}$. El índice de ajuste TLI también se encontró por sobre el punto de corte recomendado ${ }^{24}$, siendo 0,953 . El valor del RSMEA fue 0,076 , estando dentro del rango de ajuste aceptable $(0,04-0,11)^{36,37}$. El test "Close fit" no fue estadísticamente significativo $(\mathrm{p}=0,117)$, lo que significa que no es posible rechazar la hipótesis de ajuste adecuado ${ }^{28}$.

Las cargas factoriales estandarizadas fueron mayores a 0,4 , alcanzando un máximo de 0,872 para la aseveración 7. Lo anterior significa que cada una de las aseveraciones son fuertes indicadores del factor latente (Tabla 4). El mismo comentario es válido para el $\mathrm{R}^{2}$, explicando el factor latente una gran proporción de la varianza de cada aseveración.

\section{Confiabilidad}

Se calculó el Coeficiente Alfa ( $\alpha$ ) de Cronbach. El total de preguntas del cuestionario obtuvo un $\alpha=0,72$ (buena confiabilidad).

\section{Discusión}

Los talleres de simulación se han incorporado como metodología fundamental de la enseñanza de procedimientos médico-quirúrgicos. Los estudiantes valoran la oportunidad de equivocarse y aprender de sus errores en un ambiente seguro y sin riesgo para el paciente. Si bien, se ha estudiado la percepción de los estudiantes, estos estudios se han concentrado en postgrado y sin evaluación de las propiedades psicométricas. 
Tabla 4. Cargas factoriales y $\mathbf{R}^{2}$ estimadas mediante análisis confirmatorio de factores de las preguntas incluidas en la "Encuesta de percepción de los estudiantes del taller de simulación de procedimientos"

\begin{tabular}{|c|c|c|c|}
\hline Aseveraciones & $\begin{array}{l}\text { Carga } \\
\text { factorial }\end{array}$ & $\mathbf{R 2}$ & p-value \\
\hline $\begin{array}{l}\text { La práctica de modelos simulados mejora mis destrezas relacionadas con la realización de } \\
\text { este procedimiento }\end{array}$ & 0,657 & 0,432 & $<0,001$ \\
\hline Recibí retroalimentación educacional útil en la(s) sesión(es) de entrenamiento & 0,697 & 0,486 & $<0,001$ \\
\hline $\begin{array}{l}\text { La práctico con el modelo permite cometer errores que probablemente sucedan en el } \\
\text { ambiente clínico real }\end{array}$ & 0,469 & 0,220 & $<0,01$ \\
\hline El modelo usado en el taller simula o representa el procedimiento de manera realista & 0,720 & 0,518 & $<0,001$ \\
\hline $\begin{array}{l}\text { La práctica de procedimientos con el modelo simulado refuerza mi confianza en relación } \\
\text { a mis destrezas clínicas }\end{array}$ & 0,740 & 0,547 & $<0,001$ \\
\hline $\begin{array}{l}\text { Los talleres prácticos, usando modelos de procedimientos simulados, deberían ser un } \\
\text { componente obligatorio en la enseñanza de Medicina }\end{array}$ & 0,803 & 0,645 & $<0,001$ \\
\hline $\begin{array}{l}\text { La práctica con modelos de simulación me ayuda a prepararme para realizar procedimien- } \\
\text { tos clínicos de mejor manera que sólo con la experiencia clínica exclusiva }\end{array}$ & 0,872 & 0,760 & $<0,001$ \\
\hline
\end{tabular}

\section{Construcción y estimación de validez/ confiabilidad del instrumento}

Se adaptó una encuesta para pregrado. Se analizó la validez de constructo y consistencia interna ${ }^{29,30}$ de la encuesta. El análisis factorial permitió determinar el grado en que las aseveraciones del instrumento representaron al "constructo" medido ${ }^{38}$. La encuesta tiene un carácter unidimensional, sin dominios. Cada aseveración, exceptuando la número 3 "La práctica con el modelo permite cometer errores que probablemente sucedan en el ambiente clínico real", fueron fuertes indicadores del factor. Esto se contrasta con los resultados de Barsuk ${ }^{22}$, ya que los estudiantes encuestados tienen menos experiencias clínicas. Este enfrentamiento a la práctica es un elemento diferenciador en el postgrado, y puede ser un factor determinante al momento de correlacionar los posibles errores de ambos escenarios en la encuesta. Otros factores importantes para el análisis son las características de los escenarios de simulación y de los tutores expertos, las cuales podrían influir en la percepción de los estudiantes al momento de evaluar el realismo del taller (Aseveraciones 3 y 4 ) y utilidad del feedback entregado (Aseveración 2).

La consistencia interna alcanzó un valor de $\alpha=0,72$ para el total de aseveraciones, encontrándose dentro del rango considerado bueno $^{29,30}$.

\section{Aplicación del instrumento en talleres de simulación}

La encuesta se aplicó en 6 talleres de simulación. Destacó la mayor variabilidad de los ítems: 3 (La práctica con el modelo permite cometer errores que probablemente sucedan en el ambiente clínico real) y 5 (La práctica de procedimientos con el modelo simulado refuerza mi confianza en relación a mis destrezas clínicas). Esto también puede ser explicado por la menor experiencia clínica de los estudiantes de pregrado en comparación con postgrado, lo que se constata en las puntuaciones de otros ítems que no se centran en la comparación con la realidad clínica. Tal es el caso de la aseveración 1 (La práctica de modelos simulados mejora mis destrezas relacionadas con la realización de este procedimiento) y 6 (Los talleres prácticos, usando modelos de procedimientos simulados, deberían ser un componente obligatorio en la enseñanza de Medicina), los que se centran en la realización del procedimiento en simulación. A pesar de que los estudios reportados se centran en postgrado ${ }^{1}$, se destaca que los estudiantes con menor experiencia generan mayores complicaciones en los pacientes, existiendo un beneficio directo de la simulación como estrategia para evitar estos errores durante el pregrado ${ }^{39,40}$.

En todos los ítems hubo diferencias significativas entre dos o más de sus respuestas, con excepción del ítem 4 (El modelo usado en el taller simula 
o representa el procedimiento de manera realista) el cual se distribuyó casi equitativamente entre las dos más altas respuestas. Lo anterior, sumado a que las medianas del puntaje global de cada taller son elevadas, no observando variabilidad significativa, evidencia que los estudiantes valoraron muy positivamente los talleres de simulación.

Como conclusión, este estudio nos permite tener un instrumento con resultados válidos y confiables para evaluar la percepción de los estudiantes de pregrado de Medicina sobre su experiencia de aprendizaje en programas de entrenamientos de procedimientos médico-quirúrgicos por simulación. En el análisis de la encuesta se observan resultados positivos y percepciones favorables de los estudiantes frente a estos métodos de entrenamiento y se proyectan posibles relaciones entre la experiencia clínica del alumno y la percepción del aprendizaje de éste en un escenario simulado.

\section{Referencias}

1. Barsuk JH, Cohen ER, Williams MV, Scher J, Feinglass J, McGaghie WC, et al. The effect of simulation-based mastery learning on thoracentesis referral patterns. Journal of Hospital Medicine [Internet] 2016; 11 (11): 792-5. Available from: http://www.journalofhospitalmedicine.com/jhospmed/article/127995/thoracentesis-referral

2. Cisternas M, Rivera S, Sirhan M, Thone N, Valdés C, Pertuzé J, et al. Reforma curricular de la carrera de Medicina de la Pontificia Universidad Católica de Chile. Rev Med Chile [Internet] 2016; 144 (1): 102-7. Available from: http://www.scielo.cl/scielo.php?script=sci_arttext\&pid=S0034-98872016000100013\&lng=en\&nrm =iso\&tlng=en

3. Brydges R, Hatala R, Zendejas B, Erwin PJ, Cook DA. Linking Simulation-Based Educational Assessments and Patient-Related Outcomes. Academic Medicine [Internet] 2015; 90 (2): 246-56. Available from: https://insights.ovid.com/crossref?an=00001888-201502000-00032

4. Corvetto M, Bravo MP, Montaña R, Utili F, Escudero E, Boza C, et al. Simulación en educación médica: una sinopsis. Rev Med Chile [Internet] 2013; 141 (1): 70-9. Available from: http://www.scielo.cl/scielo.php?script=sci_arttext\&pid=S0034-98872013000100010\&lng=en\&n$\mathrm{rm}=\mathrm{iso} \& \operatorname{tlng}=\mathrm{en}$

5. Barsuk JH, Cohen ER, Feinglass J, Kozmic SE, McGaghie WC, Ganger D, et al. Cost Savings of Performing Paracentesis Procedures at the Bedside After Simulation-ba- sed Education. Simulation in Healthcare: The Journal of the Society for Simulation in Healthcare [Internet]. 2014; 9 (5): 312-8. Available from: https://insights.ovid. $\mathrm{com} /$ crossref?an=01266021-201410000-00006.

6. Aggarwal R, Darzi A. Simulation to Enhance Patient Safety. Chest [Internet] 2011; 140 (4): 854-8. Available from: http://linkinghub.elsevier.com/retrieve/pii/ S0012369211605205.

7. Hart D, Bond W, Siegelman JN, Miller D, Cassara M, Barker L, et al. Simulation for Assessment of Milestones in Emergency Medicine Residents. Cloutier R, editor. Academic Emergency Medicine [Internet] 2018; 25 (2): 205-20. Available from: http://doi.wiley.com/10.1111/ acem.13296.

8. Keskitalo T, Ruokamo H, Gaba D. Towards meaningful simulation-based learning with medical students and junior physicians. Medical Teacher [Internet] 2014; 36 (3): 230-9. Available from: http://www.tandfonline.com/ doi/full/10.3109/0142159X.2013.853116.

9. McGaghie WC, Issenberg SB, Cohen ER, Barsuk JH, Wayne DB. Does Simulation-Based Medical Education With Deliberate Practice Yield Better Results Than Traditional Clinical Education? A Meta-Analytic Comparative Review of the Evidence. Academic Medicine [Internet] 2011; 86 (6): 706-11. Available from: https://insights.ovid.com/crossref?an=00001888-201106000-00018.

10. Griswold S, Ponnuru S, Nishisaki A, Szyld D, Davenport M, Deutsch ES, et al. The Emerging Role of Simulation Education to Achieve Patient Safety. Pediatric Clinics of North America [Internet] 2012; 59 (6): 1329-40. Available from: http://linkinghub.elsevier.com/retrieve/pii/ S0031395512001393.

11. Boza C, León F, Buckel E, Riquelme A, Crovari F, Martínez J, et al. Simulation-trained junior residents perform better than general surgeons on advanced laparoscopic cases. Surgical Endoscopy [Internet] 2017; 31 (1): 13541. Available from: http://link.springer.com/10.1007/ s00464-016-4942-6.

12. Alvarado IJ, Henríquez R JP, Castillo RR, Sosa BJ, León FF, Varas CJ, et al. Programa pionero de simulación en sutura para estudiantes de medicina de pregrado. Revista chilena de cirugía [Internet] 2015; 67 (5): 480-5. Available from: http://www.scielo.cl/scielo.php?script=sci_arttext\&pid=S0718-40262015000500004\&lng=en\&n$\mathrm{rm}=$ iso\&tlng=en.

13. Bagnasco A, Pagnucci N, Tolotti A, Rosa F, Torre G, Sasso L. The role of simulation in developing communication and gestural skills in medical students. BMC Medical Education [Internet] 2014; 14 (1): 106. Available from: http://bmcmededuc.biomedcentral.com/ articles/10.1186/1472-6920-14-106. 
14. Graber MA. Does Simulator Training for Medical Students Change Patient Opinions and Attitudes toward Medical Student Procedures in the Emergency Department? Academic Emergency Medicine [Internet] 2005; 12 (7): 635-9. Available from: http://doi.wiley. com/10.1197/j.aem.2005.01.009.

15. Orzech N, Palter VN, Reznick RK, Aggarwal R, Grantcharov TP. A Comparison of 2 Ex Vivo Training Curricula for Advanced Laparoscopic Skills. Annals of Surgery [Internet] 2012; 255 (5): 833-9. Available from: https://insights.ovid.com/crossref?an=00000658-201205000-00005.

16. Scott DJ, Ritter EM, Tesfay ST, Pimentel EA, Nagji A, Fried GM. Certification pass rate of $100 \%$ for fundamentals of laparoscopic surgery skills after proficiency-based training. Surgical Endoscopy [Internet] 2008; 22 (8): 1887-93. Available from: http://link.springer. com/10.1007/s00464-008-9745-y.

17. Vassiliou MC, Dunkin BJ, Marks JM, Fried GM. FLS and FES: Comprehensive Models of Training and Assessment. Surgical Clinics of North America [Internet]. 2010; 90 (3): 535-58. Available from: http://linkinghub. elsevier.com/retrieve/pii/S0039610910000174.

18. Shaharan S. Evaluation of surgical training in the era of simulation. World Journal of Gastrointestinal Endoscopy [Internet] 2014; 6 (9): 436. Available from: http:// www.wjgnet.com/1948-5190/full/v6/i9/436.htm.

19. Wayne DB, Butter J, Siddall VJ, Fudala MJ, Wade LD, Feinglass J, et al. Mastery learning of advanced cardiac life support skills by internal medicine residents using simulation technology and deliberate practice. Journal of General Internal Medicine [Internet] 2006; 21 (3): 251-6. Available from: http://link.springer.com/10.1111/ j.1525-1497.2006.00341.x.

20. Wayne DB, Barsuk JH, O’Leary KJ, Fudala MJ, McGaghie WC. Mastery learning of thoracentesis skills by internal medicine residents using simulation technology and deliberate practice. Journal of Hospital Medicine [Internet] 2008; 3 (1): 48-54. Available from: http:// www.journalofhospitalmedicine.com/jhospmed/article/126959/mastery-learning-procedural-skills.

21. Barsuk JH, Ahya SN, Cohen ER, McGaghie WC, Wayne DB. Mastery Learning of Temporary Hemodialysis Catheter Insertion by Nephrology Fellows Using Simulation Technology and Deliberate Practice. American Journal of Kidney Diseases [Internet] 2009; 54 (1): 70-6. Available from: http://linkinghub.elsevier.com/retrieve/ pii/S0272638609004211.

22. Barsuk JH, Cohen ER, Vozenilek JA, O'Connor LM, McGaghie WC, Wayne DB. Simulation-Based Education with Mastery Learning Improves Paracentesis Skills.
Journal of Graduate Medical Education [Internet] 2012; 4 (1): 23-7. Available from: http://www.jgme.org/doi/ abs/10.4300/JGME-D-11-00161.1.

23. Hasson F, Keeney S, McKenna H. Research guidelines for the Delphi survey technique. Journal of Advanced Nursing [Internet] 2000; 32 (4): 1008-15. Available from: http://doi.wiley.com/10.1046/j.1365-2648.2000. t01-1-01567.x

24. MacCallum RC, Browne MW, Sugawara HM. Power analysis and determination of sample size for covariance structure modeling of fit involving a particular measure of model. Psychological Methods [Internet] 1996; 13 (2): 130-49. Available from: http://doi.apa.org/getdoi. cfm?doi=10.1037/1082-989X.1.2.130.

25. MacCallum RC, Browne MW, Cai L. Testing differences between nested covariance structure models: Power analysis and null hypotheses. Psychological Methods [Internet] 2006; 11 (1): 19-35. Available from: http:// doi.apa.org/getdoi.cfm?doi=10.1037/1082-989X.11.1.19

26. MacCallum R, Lee T, Browne MW. The Issue of Isopower in Power Analysis for Tests of Structural Equation Models. Structural Equation Modeling: A Multidisciplinary Journal [Internet] 2010; 17 (1): 2341. Available from: http://www.tandfonline.com/doi/ abs/10.1080/10705510903438906.

27. Brown BL, Hendrix SB, Hedges DW, Smith TB. Multivariate Analysis for the Biobehavioral and Social Sciences [Internet]. Hoboken, NJ, USA: John Wiley \& Sons, Inc.; 2011. Available from: http://doi.wiley. com/10.1002/9781118131626.

28. Wang J, Wang X. Structural Equation Modeling: Applications Using Mplus. First. United Kingdom: Jhon Wiley \& Sons, Inc; 2012. 478 p.

29. Heo M, Kim N, Faith MS. Statistical power as a function of Cronbach alpha of instrument questionnaire items. BMC Medical Research Methodology [Internet] 2015; 15 (1): 86. Available from: http://bmcmedresmethodol. biomedcentral.com/articles/10.1186/s12874-015-0070-6.

30. Cronbach LJ. Coefficient alpha and the internal structure of tests ${ }^{\star}$ lf $\sim$ j. cronbach 1951; 16 (3): 297-8.

31. Kolenikov S, Angeles G. The Use of Discrete Data in PCA: Theory, Simulations, and Applications to Socioeconomic Indices. Chapel Hill: Carolina Population Center, University of North Carolina 2004; 1-59.

32. Kaiser HF. An index of factorial simplicity 1974; 39 (1): 31-6.

33. Acock AC. Discovering Structural Equation Modeling Using Stata. 1st ed. Press S, editor. 2015. 306 p.

34. $\mathrm{Hu} \mathrm{L}$, Bentler PM. Cutoff criteria for fit indexes in covariance structure analysis: Conventional criteria versus new alternatives. Structural Equation Modeling: 
A Multidisciplinary Journal [Internet] 1999; 6 (1): 1-55. Available from: http://www.tandfonline.com/doi/ abs/10.1080/10705519909540118.

35. Bentler PM, Bonett DG. Significance tests and goodness of fit in the analysis of covariance structures. Psychological Bulletin [Internet] 1980; 88 (3): 588-606. Available from: http://content.apa.org/journals/bul/88/3/588.

36. Byrne BM. Structural Equation Modeling with LISREL, PRELIS, and SIMPLIS: Basic Concepts, Applications, and Programming. New Jersey: Lawrence Erlbaum Associates, Inc; 1998. 432 p.

37. Byrne BM. Structural Equation Modeling with Mplus: Basic Concepts, Applications, and Programming. New York: Routledge, Taylor and Francis Group; 2012.

38. Bitran M, Mena B, Riquelme A, Padilla O, Sánchez I,
Moreno R. Desarrollo y validación de un instrumento en Español para evaluar el desempeño de docentes clínicos a través de las percepciones de sus estudiantes. Rev Med Chile 2010; 138 (6): 685-93.

39. Barsuk JH, Cohen ER, Williams MV, Scher J, Jones SF, Feinglass J, et al. Simulation-Based Mastery Learning for Thoracentesis Skills Improves Patient Outcomes. Academic Medicine [Internet] 2017; 1. Available from: http://insights.ovid.com/crossref?an=00001888-900000000-98076.

40. Sharzehi K, Jain V, Naveed A, Schreibman I. Hemorrhagic Complications of Paracentesis: A Systematic Review of the Literature. Gastroenterology Research and Practice [Internet] 2014; 2014: 1-6. Available from: http://www.hindawi.com/journals/grp/2014/985141/ 